\title{
Evolution of Non-Equilibrium Profile in Adsorbate Layer under Compressive Strain
}

\author{
Enzo Granato · S.C. Ying \\ Received: date / Accepted: date
}

\begin{abstract}
We investigate the time evolution of an initial step profile separating a bare substrate region from the rest of the compressively strained adsorbate layer near a commensurate to incommensurate transition. The rate of profile evolution as a function of the mismatch, coverage and the strength of the substrate potential are determined by Brownian molecular dynamics simulations. We find that the results are qualitatively similar to those observed for the $\mathrm{Pb} / \mathrm{Si}(111)$ system. The anomalously fast time evolution and sharpness of the non-equilibrium profile can be understood through the domain wall creation at the boundary and its subsequent diffusion into the interior of the adsorbate layer.
\end{abstract}

Keywords Nanotribology · Friction mechanisms · Dynamic modeling

PACS 68.43.Jk $\cdot 68.43 . \mathrm{De} \cdot 64.70 . \mathrm{Rh}$

\section{Introduction}

The system of an adsorbate layer with a lattice mismatch to the underlying substrate has been extensively studied both from the experimental and theoretical side. In the monolayer regime, it provides a realization of many two dimensional phases 1 and has also been used as a simple model for studying sliding friction

\section{Enzo Granato}

Laboratório Associado de Sensores e Materiais, Instituto Nacional de Pesquisas Espaciais,

12227-010 São José dos Campos, São Paulo, Brazil

\section{S.C. Ying}

Department of Physics, Brown University,

Providence, Rhode Island 02912, USA

E-mail: seechen.y@gmail.com phenomena on surfaces 2, 3, 4,5. In particular, the rich phenomena of commensurate to incommensurate phase transition depends on the competition between the adsorption energy and the strain energy from the lattice mismatch. Recently, Tringides et al. 6, 7, have done an extensive study of the $\mathrm{Pb} / \mathrm{Si}(111)$ system and found the "Devil's Stair Case" within a narrow range of coverage as predicted by the theory. For a larger coverage, they have further found that the adsorbate layer exhibited anomalous mass transport through the time evolution of step profile created by desorbing the adatoms in a small region. This anomalous transport is characterized by a sharp non-diffusive profile at all times as well as a critical coverage below which the rate of the profile evolution slows down exponentially with decreasing coverage. To date, there is still no detailed understanding of the microscopic origin of this anomalous mass transport. Very recently, Huang et al. 8, have used molecular dynamics simulations in a generalized onedimensional Frenkel-Kontorova model to demonstrate that collective diffusion of $\mathrm{Pb}$ atoms in the wetting layer on $\mathrm{Si}(111)$ via domain wall motion for coverage beyond monolayer can lead to fast mass transport. However, the microscopic origin of a critical coverage for the profile evolution is still not understood. In this paper, we present a study of a generic two dimensional adsorbate system [3, 4, 5] with a well understood transition from a commensurate $c(2 \times 2)$ phase to an incommensurate phase. A non-equilibrium profile is created in the adsorbate layer and the time evolution of the profile is then studied numerically via molecular dynamics simulation. In particular, we focus on the rate of profile evolution as a function of the lattice mismatch, the coverage and the strength of the substrate potential. We find that the results are qualitatively similar to those observed for the $\mathrm{Pb} / \mathrm{Si}(111)$ system. The main results can be un- 
derstood via microscopic mechanisms that are generic to an adsorbate layer under a compressive strain near a commensurate-incommensurate transition. In particular, the time evolution of the non-equilibrium profile is governed by the rate of domain wall creation at the boundary and its subsequent diffusion into the interior of the adsorbate layer.

\section{Model}

We consider a two-dimensional (2D) adsorbate layer with particle interactions modeled by the Lennard-Jones (LJ) pair potential,

$v(r)=\epsilon_{0}\left[\left(r_{o} / r\right)^{12}-2\left(r_{o} / r\right)^{6}\right]$,

with 2 parameters: minimum energy distance $r_{o}$ and amplitude $\epsilon_{o}$. The substrate is represented by a rigid sinusoidal periodic potential,

$V(x, y)=V_{0}[2-\cos (2 \pi x / a)-\cos (2 \pi y / a)]$,

with amplitude $V_{o}$ and minima on the sites of a square lattice with lattice spacing $a$. This model has been used previously in the study of nonlinear sliding friction of adsorbed overlayers [2,3,4,5]. The system consists of $5000-10000$ adatoms in an area of size $L \times L$. Periodic boundary conditions are applied. Throughout this paper, we will choose the amplitude of the substrate potential and the substrate lattice spacing as the scales for the energy and length and all quantities will be expressed in dimensionless form. In Fig. 1, we show the ground state energy of the adsorbate system at half coverage, $\theta=1 / 2$, for different values of the parameter $r_{o}$ in the LJ potential. For $r_{o}<1.56$, the adsorbate layer is in a commensurate $c(2 \times 2)$ phase with interparticle spacing of $\sqrt{2}$. The ground state energy is a minimum at $r_{0}=1.45$. Changing the parameter $r_{o}$ amounts to changing the lattice mismatch or changing the compressive strain in the commensurate phase. For $r_{0}>1.45$, the layer is compressed relative to the minimum energy configuration but still remains in the commensurate $\mathrm{c}(2 \mathrm{x} 2)$ phase as seen in the constancy of the amplitude of the structure factor at wavevector $Q=(\pi, \pi)$. For $r_{0}>1.56$, the value of the structure factor at wavevector $Q=(\pi, \pi)$ starts to decrease, indicating the entrance into an incommensurate phase.

In the next two sections, we will present results when the adsorbate atoms are removed from a circular region with radius $R<<L$ to mimic the experimental creation of a step profile [6,7] via localized desorption. We will then follow the temporal evolution of this nonequilibrium profile to explore the dynamics of this system. These studies will be performed by varying either
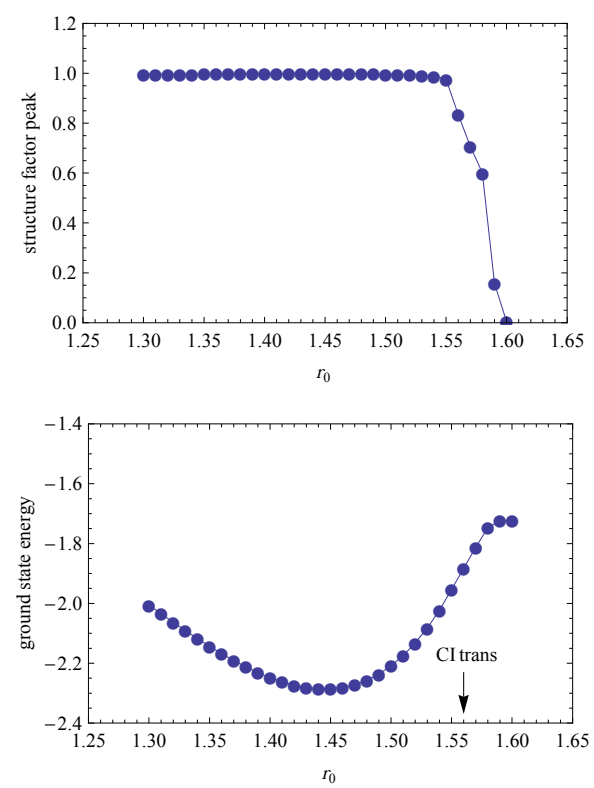

Fig. 1 Structure factor peak at wavevector $Q=(\pi, \pi)$ and ground state energy as a function of the minimum energy separation $r_{o}$ in the LJ pair potential. For coverage $\theta=0.5$.

the mismatch through the variation of $r_{0}$, the coverage or the strength of the substrate potential $V_{0}$. The calculations were performed using Brownian molecular dynamics (MD) simulations [9] with the time variable discretized in units of $\delta t=0.002-0.01 \tau_{m d}$, where $\tau_{m d}=\left(m a^{2} / V_{o}\right)^{1 / 2}$.

\section{Numerical results and discussion}

3.1 Non-equilibrium profile evolution as a function of lattice mismatch

For studying the temporal evolution of the non-equilibrium profile, the adsorbate layer is first equilibrated at finite temperature. Then, adatoms are removed from a circular region in the center of the system. The radius of the hole is small, corresponding to a removal of only 150 adatoms for the system of 5000 particles. Thus, the density of the region outside does not change significantly as the non-equilibrium profile approaches equilibrium. Configurations are stored at regular time intervals.

In Fig. 2, we show snapshots for the evolution of the non-equilibrium profile for increasing times. The parameters are $r_{o}=1.53, L=50$, temperature $T=0.4$ and coverage 0.5 . For this set of parameters, the initial equilibrium phase before the creation of the nonequilibrium profile region is the commensurate $c(2 \times 2)$ phase with interparticle spacing $\sqrt{2}$. We note that the profile remains sharp initially as distinct from the typical diffuse profile resulting from pure diffusion dynam- 


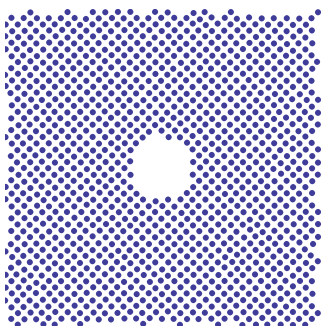

23

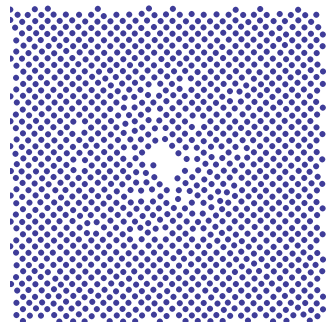

Fig. 2 Snapshots for the evolution of the non-equilibrium profile for increasing times. For $r_{o}=1.53, L=50$, temperature $T=0.4$ and coverage $\theta=0.5$. The labels correspond to times in units of $10^{4} \mathrm{MD}$ time steps.

ics. This is qualitatively similar to the experimental observation for $\mathrm{Pb} / \mathrm{Si}(111)$, although the small radius of the hole region prevents us from determining a conclusive behavior for the longtime region where the noise is significant. To study the time evolution of the profile as a function of the lattice mismatch, simulations studies were also done for different values of $r_{0}$ at half coverage. The time $\tau$ (in MD steps) that it takes to completely refill the circular region and return to the equilibrium is obtained and the average refilling velocity is given by $1 / \tau$. The number of particles inside the circular region and the radius difference $\left(R_{o}-R(t)\right)$, where $R_{o}$ is the initial radius of the hole, are both increasing functions of time. Figs 3 show the behavior of these quantities for $r_{o}=1.51$ and 1.56. Note that the time scale for the two different values of $r_{0}$ differ by more than an order of magnitude showing that the refilling dynamics speed up rapidly beyond $r_{o}=1.51$.

In Fig. 4, we plot the average refilling velocity vs $r_{0}$. There is a critical value of $r_{0} \approx 1.50$ below which the refilling velocity $(1 / \tau)$ is too small to measure in our simulation study. This value is smaller than the critical value of $r_{0} \approx 1.55$, corresponding to the bulk commensurate-incommensurate transition at the temperature $T=0.4$. Thus the initial state of the adsorbate layer is still in the commensurate $c(2 \times 2)$ phase and the diffusion dynamics in the bulk is exceedingly small. This is the first hint that the boundary effects play a significant role in the time evolution of the non-
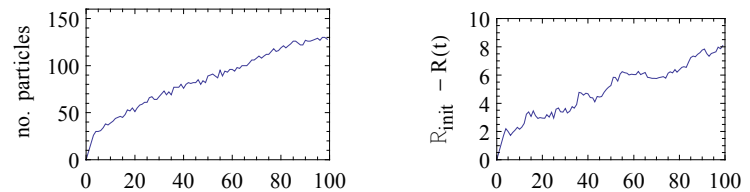

time $/\left(8 \times 10^{3}\right)$

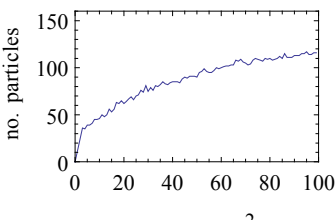

time $/\left(8 \times 10^{3}\right)$

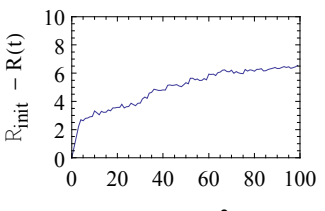

time / $\left(4 \times 10^{2}\right)$

Fig. 3 Time dependence of the number of particles inside the hole region $\left(R<R_{o}\right)$ and the radius difference $R_{o}-R(t)$, for $T=0.4, L=100$ and coverage $\theta=0.5$. For $r_{o}=1.51$ (top) and $r_{o}=1.56$ (bottom)

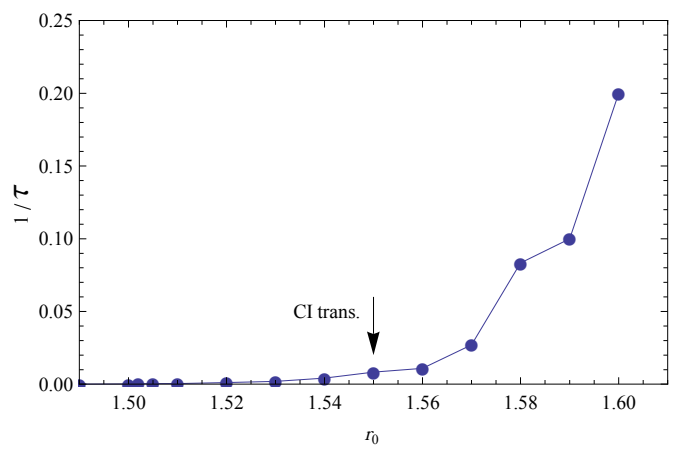

Fig. 4 Inverse refilling time $1 / \tau$ (in units of $10^{3} \mathrm{MD}$ time steps) as a function of the LJ pair potential parameter $r_{o}$. For $L=50, T=0.4$ and coverage $\theta=0.5$.

equilibrium profile. Beyond the value of $r_{0} \approx 1.56$, the refilling velocity rapidly takes off.

To understand the role of the boundary effect qualitatively, we can look at the known results of a semiinfinite one-dimensional Frenkel-Kontorova chain with an open boundary at one end 1, 10. Under a compressional strain, the last adatom is pushed out towards the next maximum in the substrate potential due to the lack of balancing force from the unoccupied site, with the neighboring adatoms making small adjustments to reach equilibrium. There is an activation barrier for the end atom to hop over to the unoccupied side and thus creating a "light" domain wall at the boundary. For increasing value of the parameter $r_{0}$, the mismatch and the compressive stress increases and the end atom gets pushed further out. Correspondingly, the activation barrier for the "light" wall decreases and eventually this barrier can vanish leading to the spontaneous generation of a "light" wall at the boundary. At finite 
temperatures, the onset of rapid time evolution of the non-equilibrium profile should correspond to the value of $r_{0}$ at which the barrier is comparable to $k_{B} T$. Once generated, this "light" wall can now move rapidly further into the chain under the mechanism of biased diffusion due to the compressive stress. The net effect is the motion of the boundary by one lattice spacing into the open area. The whole process can then repeat itself leading to the advancement of the profile and refilling of the initially empty region. Obviously, the process will stop at some point if the chain is finite. However, for a semi-infinite chain or one maintained at a constant chemical potential at the far end, the unfilled region can be filled at a steady rate via this mechanism. The creation of the "light" wall at the boundary and its rapid propagation into the interior is the key ingredient in understanding the anomalous evolution of the nonequilibrium profile as shown in our numerical study. Note that at the onset of this rapid anomalous transport, normal bulk diffusion rate of domain walls and defects are negligible in the interior of the adsorbate still in the commensurate state. However, the "light" wall created at the boundary can diffuse inwards because the unbalanced boundary stress essentially provides an inward driving force leading to a biased diffusion. For the same reason, the profile is sharp because the dynamics of its evolution is governed by a biased diffusion mechanism rather than the normal diffusive behavior as observed in typical Boltzman-Matano systems [11. The activation barrier for the "light" wall has been studied quantitatively in the 1D Frenkel-Kontorova model in the continuum limit [1, 10, but its detailed dependence on the mismatch for a fully discrete $2 \mathrm{D}$ model as presented in this study is yet unknown.

We have also studied the time evolution dynamics as a function of the strength of the substrate potential as shown in Fig. 5. As the substrate potential gets weaker, the rate of time evolution of the profile increases. This can be interpreted as due to two factors. The first is that the activation barrier of the "light" wall at the boundary decreases with decreasing strength of the substrate potential. The second factor is that the biased diffusion rate of the "light" wall towards the interior is also enhanced through the reduction of the substrate potential.

3.2 Non-equilibrium profile evolution as a function of coverage

While the variation of the LJ potential parameter $r_{0}$ and hence the lattice mismatch nicely illustrates the role that the boundary effects play in determining the

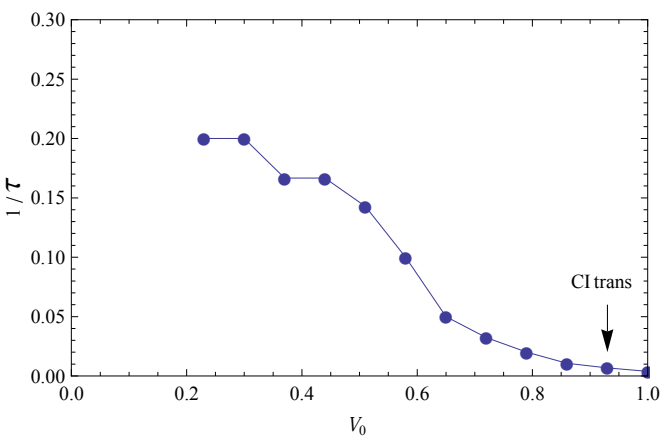

Fig. 5 Inverse refiling time $1 / \tau$ (in units of $10^{4}$ MD time steps) as a function of the strength of the substrate potential $V_{0}$. For $L=50, r_{o}=1.53, T=0.4$ and coverage $\theta=0.5$.
1

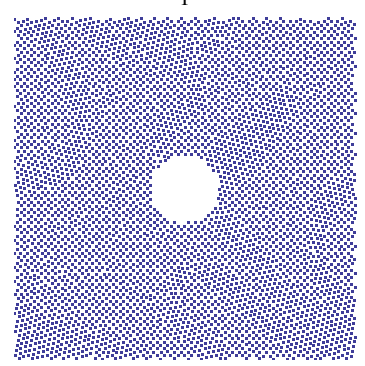

29

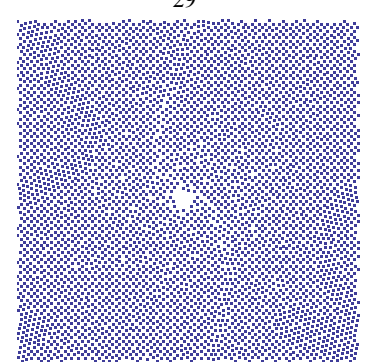

13

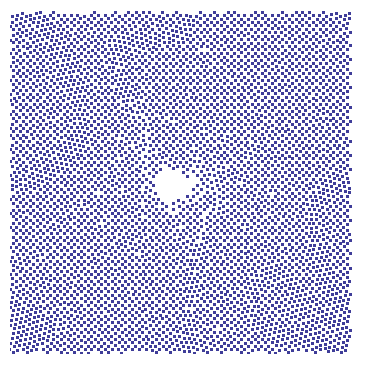

40

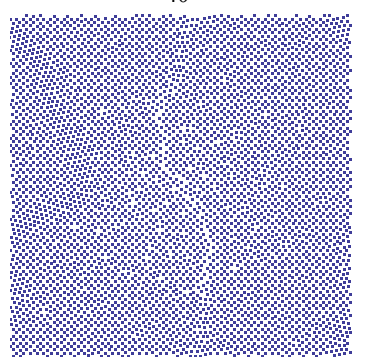

Fig. 6 Snapshots of the layer at different times during refilling of the initial hole. For $L=100, r_{o}=1.53, T=0.4$ and coverage $\theta=0.508$. The labels correspond to times in units of $3 \times 10^{4} \mathrm{MD}$ time steps.

time evolution of the non-equilibrium profile, experimentally variation of the lattice mismatch is hard to realize. Instead, the easy parameter to vary in a real system such as $\mathrm{Pb} / \mathrm{Si}(111)$ is the coverage [6, 7]. In this section, we study the effect of varying the coverage beyond the ideal commensurate coverage of $\theta=1 / 2$ on the refilling dynamics. Shown in the Figs. 6 and 7 are the results of the refilling dynamics as a function of the coverage for a fixed value of potential parameter $r_{0}=1.53$.

As shown in Fig. 7, there is a critical coverage of $\theta \approx 0.5$ at $\mathrm{T}=0.4$, below which the refilling dynamics is too slow to measure in our study. Beyond a value of $\theta \approx 0.54$ the refilling dynamics rapidly take off. Qualitatively, the increase of coverage beyond $\theta=1 / 2$ again 


\section{Conclusion}

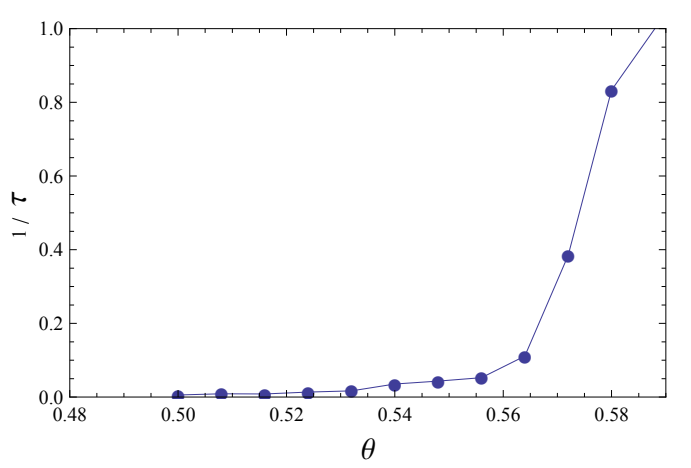

Fig. 7 Inverse refiling time $1 / \tau$ (in units of $10^{4} \mathrm{MD}$ time steps) as a function of coverage $\theta$ for $L=100, r_{o}=1.53$ at $T=0.4$.

increases the compressive strain and facilitates the creation of a light wall at the boundary, as discussed in the last section. However, in this case another factor come into consideration in determining the refilling dynamics. Beyond the half coverage $\theta=1 / 2$, the configuration in the bulk is now incommensurate and there are either defects or domain walls (heavy type) to accomodate the extra coverage. For a system below the Aubry transition [12,13, 14], these defects are pinned and immobile. In this phase, the diffusion of the "light" wall created at the boundary into the interior will be blocked by these defects and the refilling process will stop. As the coverage increases, the critical potential strength for the Aubry transition decreases [12] and these defects and domain walls become mobile. At this point, the refilling dynamics can proceed smoothly just as discussed in the last section where only the value of the mismatch is varied. Thus, when the coverage is increased beyond the ideal commensurate value of $\theta=1 / 2$, the time evolution of the profile is determined by the convolution of both the activation rate of domain walls at the boundary and the diffusion rate of defects in the interior of the adsorbate layer. We like to point out that our use of the term "critical coverage" in the present context differ somewhat from what was used in the empirical description of the data for $\mathrm{Pb} / \mathrm{Si}(111)$ [6, 7] where the critical coverage was used to describe the value of the coverage where the rate crossover from a region with an activation barrier increasing with decreasing coverage to another region with weak coverage dependence. In particular, the details of the rapid increase of the profile evolution rate beyond the critical coverage has not been investigated experimentally.
In this paper we have investigated the time evolution of non-equilibrium profile in an adsorbate system near a commensurate to incommensurate transition. In the commensurate $c(2 \times 2)$ phase, the mobility and diffusive rate for the bulk system is essentially zero. However if the absorbate system is under compressive strain due to lattice mismatch with the substrate, then the barrier for activation of a "light" domain wall is reduced at the boundary separating the adsorbate from the unoccupied region. For increasing strain energy due to either increased lattice mismatch or increase of coverage beyond the ideal coverage for the commensurate $c(2 \times 2)$ phase, the barrier for this boundary domain wall generation will be lowered to the point where it can be easily activated thermally. The profile can then evolve in time through the repeated activation of the boundary domain wall and its biased diffusion into the interior of the adsorbate layer. This mechanism is similar to the one proposed by Huang et al. [8]. The difference is that in our study the coverage dependence of the activation barrier of the boundary domain walls comes out naturally as a consequence of the microscopic model and is not invoked as an additional assumption. The profile remains sharp during its evolution because it is continuously being driven by the unbalanced compressive stress at the boundary. When the stress at the boundary is increased via increasing the coverage beyond the ideal commensurate coverage instead of changing the lattice mismatch, an extra ingredient is required besides the activation of the boundary domain wall. In this case, the interior of the adsorbate layer has intrinsic defects or domain walls to accommodate the extra adatoms. Depending on the substrate potential and the coverage, these defects can be either pinned or depinned. The transition between these two phases correspond to the "Aubry transition " well studied in the literature [12, 13.14. When the domain walls are "pinned", the propagation of the boundary generated domain wall into the interior will be blocked by the pinned domain walls and the time evolution of the non-equilibrium profile will also stop. The anomalous fast transport can only be achieved when the system is in the unpinned side of the incommensurate phase. The transition to this side of the "Aubry phase" requires the coverage to exceed a critical value, leading to an extra requirement for the the rapid propagation of the non-equilibrium profile besides the activation of the boundary domain wall. This aspect also differs from the generalized onedimensional Frenkel-Kontorova model results of Huang et al. 8, where domain walls in the bulk is always mobile and unpinned beyond the monolayer coverage. Al- 
though the present study is for a specific model system with a relatively simple commensurate-incommensurate phase transition, the generic features of the profile evolution is applicable to a wide class of adsorbate layer under compressive strain. The mechanism of anomalous mass transport via activation and propagation of boundary domain walls should play an important role in tribological systems.

Acknowledgements We acknowledge helpful discussions with M.C. Tringides, M.S. Altman C.Z. Wang, T. Ala-Nissila and C. Zdenek. E.G. was supported by Fundação de Amparo à Pesquisa do Estado de São Paulo - FAPESP (Grant No. 07/08492-9) and in part by computer facilities from Centro Nacional de Processamento de Alto Desempenho - CENAPAD$\mathrm{SP}$

\section{References}

1. Pokrovsky, V.L., Talapov, A.L.: Theory of Incommensurate Crystals. Harwood Academic Publishers. New York (1984).

2. Persson, B.N.J.: Theory and simulation of sliding friction. Phys. Rev. Lett. 71, 1212-1215 (1993)

3. Persson, B.N.J.: Sliding Friction: Physical Principles and Applications. Springer, Heidelberg (1998)

4. Granato, E., Ying, S.C.: Transverse Thermal Depinning and Nonlinear Sliding Friction of an Adsorbed Monolayer. Phys. Rev. Lett. 85, 5368-5371 (2000)

5. Granato, E., Ying, S.C.: Nonlinear sliding friction of adsorbed overlayers on disordered substrates. Phys. Rev. B 69, $125403(2004)$

6. Man, K.L., Tringides, M.C., Loy, M.M.T., Altman, M.S.: Anomalous Mass Transport in the $\mathrm{Pb}$ Wetting Layer on the Si(111) Surface. Phys. Rev. Lett. 101, 226102 (2008)

7. Tringides, M.C., Hupalo, M., Man, K.L., Loy, M.M.T., Altman, M.S.: Wetting Layer Super-Diffusive Motion and QSE Growth in Pb/Si. In: Michailov, M. (ed.) Nanophenomena at Surfaces, pp. 39-65. Springer, Heidelberg (2011)

8. Huang, Li, Wang, C.Z., Li, M.Z., Ho, K.M.: Coveragedependent Colletive Ciffusion of Dense Pd Wetting Layer on Si(111). Phys. Rev. Lett. 108, 026101 (2012)

9. Allen, M.P., Tildesley, D.J.: Computer Simulation of Liquids. Clarendon, Oxford (1987)

10. Frank F.C., Van der Merwe, J.H.: One-Dimensional Dislocations. I. Static Theory. Proc. R. Soc. A 198, 205-216 (1949)

11. Loburets, A.T., Naumovets, A.G., Vedula, Yu.S.: Diffusion of dysprosium on the (112) surface of molybdenum. Surface Science 399, 297-304 (1998)

12. Floria, L.M., Mazo, J.J.: Dissipative dynamics of the Frenkel-Kontorova model. Adv. Phys. 45, 505-598 (1996)

13. Coppersmith, S.N., Fisher, D.S.: Pinning transition of the discrete sine-Gordon equation. Phys. Rev. B 28, 2566-2581 (1983)

14. Peyard, M., Aubry, S.: Critical behaviour at the transition by breaking of analyticity in the discrete FrenkelKontorova model. J. Phys. C 16, 1593 (1983) 